\title{
Studies of missing energy decays of B meson at Belle II
}

\author{
Elisa Manoni*广 \\ Istituto Nazionale di Fisica Nucleare, Sezione di Perugia \\ E-mail: elisa.manoni@pg.infn.it
}

The Belle II experiment at the SuperKEKB collider is a major upgrade of the KEK "B factory" facility in Tsukuba, Japan. The machine is designed for an instantaneous luminosity of $8 \times$ $10^{35} \mathrm{~cm}^{-2} \mathrm{~s}^{-1}$, and the experiment is expected to accumulate a data sample of about $50 \mathrm{ab}^{-1}$ in five years of running. With this amount of data, decays sensitive to physics beyond the Standard Model can be searched for with unprecedented sensitivity. One promising set of modes is physics processes with missing energy such as $B^{+} \rightarrow \tau^{+} v, B \rightarrow D^{(*)} \tau \nu$, and $B \rightarrow K^{(*)} v \bar{v}$ decays.

The $B \rightarrow K^{(*)} v \bar{v}$ decay provides one of the cleanest experimental probes of the flavour-changing neutral current process $b \rightarrow s v \bar{v}$, which is sensitive to physics beyond the Standard Model. However, the missing energies of the two neutrinos in the final state make the measurement challenging and require full reconstruction of the spectator $B$ meson in $e^{+} e^{-} \rightarrow \Upsilon(4 S) \rightarrow B \bar{B}$ events. Observation of the $B \rightarrow K^{(*)} v \bar{v}$ decay will become possible with the large data set to be collected by the upgraded Belle II experiment running at the SuperKEKB accelerator in Japan. A challenge of this analysis will be understanding and suppressing backgrounds. This talk discusses such backgrounds and the expected sensitivity of Belle II for this rare decay.

The European Physical Society Conference on High Energy Physics

5-12 July, 2017

Venice

* Speaker.

${ }^{\dagger}$ On behalf of the Belle II collaboration. 


\section{Strategies for new physics searches in $B$ final states with missing energy}

Searches for physics beyond the Standard Model (SM) can be performed at accelerator experiments both at the energy and at the intensity frontier. In the first case, states predicted by New Physics (NP) models are searched for as real particles, making this approach limited by the energy of the beam(s). In the second case, NP is searched for by the virtual contribution of any non-standard particles. In such case, high-statistics samples are needed to observe deviations from the SM predictions. In the intensity frontier context, the Belle II experiment, at the SuperKEKB accelerator in Japan, will search for NP effects by exploiting a plethora of $B, D, \tau$, and $\Upsilon$ decay modes, with $50 \mathrm{ab}^{-1}$ collected in 5 years of data taking. With respect to its predecessors, BaBar and Belle, the detector will have to work in a higher luminosity environment (factor of 40 with respect to Belle) resulting in a higher statistic sample but also higher rate of radiation hitting the detector components coming from beam-beam, intra-beam, and beam-material interactions. Such processes are referred to as "machine background". In order to cope with the higher backgrounds and maintain the performance achieved by Belle, an upgrade of the detector is ongoing. Data taking is foreseen to start in the first half of 2018. In $e^{+} e^{-}$collisions at the $\Upsilon(4 S)$ mass, final states containing only charged and neutral $B \bar{B}$ pairs will be produced. Thanks to the clean environment due to a well-defined initial state, a good and efficient reconstruction of decays with both charged and neutral particles, and a full-solid-angle detector which ensure high detector hermeticity, the Belle II experiment is an ideal environment to search for decays with missing energy in the final state in which NP states can contribute both as intermediate particles and as new sources of missing energy. One of the experimental methods used for this purpose is called Full Event Interpretation (FEI) in which one of the $B$ 's is reconstructed in semileptonic or hadronic modes (tag side) and on the other side the signal signature (signal side) is searched for. The FEI algorithm, already in use at Belle, has been improved both in terms of the reconstruction algorithm and of the number of reconstructed decay modes, resulting in a factor of 2 improvement in efficiency with respect to the Belle performance. An overview on the Belle II studies for $B \rightarrow D^{(*)} \tau \nu, B \rightarrow \tau \nu$, and $B \rightarrow K^{(*)} v \bar{v}$ will be described in the following.

\section{Search for $B \rightarrow D^{(*)} \tau \nu$}

The $B \rightarrow D^{(*)} \tau \nu$ transition is a tree-level process mediated, in the SM, by the $W$ boson. NP scenarios predict the existence of a new charged boson, e.g. a charged Higgs, that can replace the $W$. Deviations from the SM expectation for the ratio of branching fracstions $R\left(D^{(*)}\right)=\frac{\mathscr{B}\left(B \rightarrow \bar{D}^{(*)} \tau^{+} v\right)}{\mathscr{B}\left(B \rightarrow \bar{D}^{(*)} \ell \bar{v}\right)}$, with $\ell=e, \mu$, have been searched for by the BaBar, Belle, and LHCb collaborations. The experimental results for $R_{D^{*}}$, along with the SM expectations [1], are summarised in the left plot of Fig. 1 . In the $R_{D^{*}} R_{D}$ plane, the most recent experimental world average is $4.1 \sigma$ away from the SM expectations. The current measurements are statistically limited, while the dominant systematic uncertainties arise from the limited signal MC samples and the limited knowledge of dominant backgrounds (involving soft pions produced in the $D^{*}$ decay). Large signal MC samples and dedicated measurement for background characterisation with large data samples, along with improved tracking algorithm to recover efficiency in the low momentum range, are foreseen at Belle II. Studies of the $q^{2}$ distributions and precise measurements of the $\tau$ polarisation, both essential to distinguish 

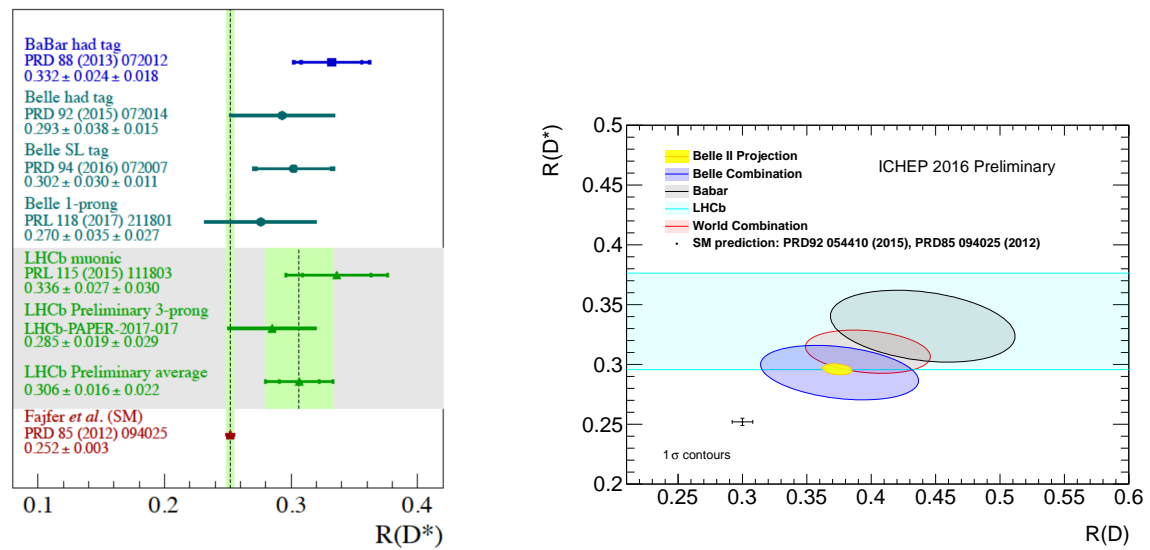

Figure 1: Left: experimental results and SM expectations for $R\left(D^{*}\right)$. Right: expected Belle II constraints on $R_{D}$ and $R_{D^{*}}$ compared to existing experimental constraints, as of ICHEP 2016, and SM prediction.

among NP models, will also be feasible. Extrapolating the existing BaBar and Belle results, the following precision with the full Belle II statistics is expected: $\frac{\sigma\left(R_{D}\right)}{R_{D}}=2.0 \%$ (stat.) $\pm 2.5 \%$ (syst.), $\frac{\sigma\left(R_{D^{*}}\right)}{R_{D^{*}}}=1.0 \%$ (stat.) $\pm 2.0 \%$ (syst.). The expected Belle II constraints on $R_{D}$ and $R_{D^{*}}$, compared to existing experimental measurements as of ICHEP 2016 and to the SM predictions, are shown in the right plot of Fig. 1.

\section{Search fo $B \rightarrow \tau v$}

Similarly to $B \rightarrow D^{(*)} \tau \nu, B \rightarrow \tau \nu$ decay is also a tree-level process mediated in the SM by a $W$, which can be replaced by non-standard charged bosons. The branching fraction of the purely leptonic decay depends on the CKM element $\left|V_{u b}\right|$ and on the form factor $f_{B}$. Using the values in Ref. [3], the branching ratio SM prediction is $\mathscr{B}(B \rightarrow \tau v)=(0.77 \pm 0.06) \times 10^{-4}$. This has to be compared to the BaBar and Belle world average $\mathscr{B}(B \rightarrow \tau v)=(1.06 \pm 0.19) \times 10^{-4}$, obtained by using both hadronic and semileptonic tag [4]. Combining the available results, the branching fraction measurement exceeds the $5 \sigma$ significance. Moreover, the world average is consistent with the SM expectation at the $2 \sigma$ level. The measurements are statistically limited. The dominant systematic effects are due to disagrement between data and simulation and to the estimation of the parameterisation of signal and background shape in the fit for the yield extraction. Some of them are statistical in origin. An analysis using the Belle II full simulation, with the hadronic B reconstruction technique, has been performed. Extrapolating the systematic effects from the most recent Belle analyses and considering the full Belle II statistics, uncertainties of the order of $6 \%$ on the branching fraction are expected from both the hadronic and the semilptonic analysis.

\section{Search fo $B \rightarrow K^{(*)} v \bar{v}$}

The $B \rightarrow K^{(*)} v \bar{v}$ decay proceeds through a flavour-changing neutral current, prohibited at tree level in the SM. NP contributions (from new mediators in the box or in the penguin diagrams or due to new sources of missing energy) may be comparable to the SM ones. The process is also 


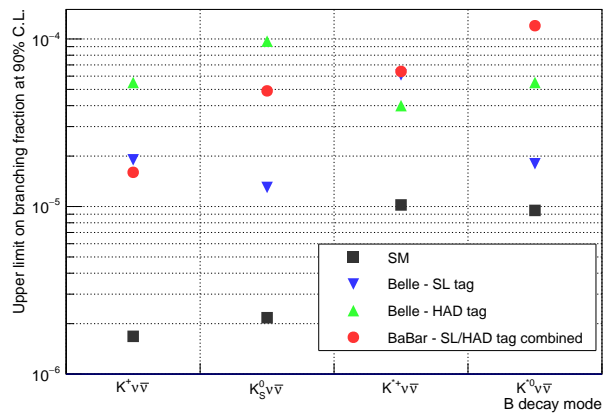

\begin{tabular}{ll|c}
\hline & "BGx0" & "BGx1" \\
\hline$N_{b k g}$ & $6415 \pm 80$ & $3678 \pm 61$ \\
$\varepsilon\left(10^{-4}\right)$ & $10.3 \pm 0.3$ & $5.38 \pm 0.23$ \\
$N_{s i g} / \sqrt{N_{b k g}}$ & 0.16 & 0.15 \\
$\mathrm{UL}\left(10^{-4}\right)$ & 2.6 & 3.8 \\
\hline
\end{tabular}

Figure 2: Left: obtained upper limit and expected branching fraction in the SM for $B \rightarrow K^{(*)} v \bar{v}$. Right: number of generic events $\left(N_{b k g}\right)$, signal selection efficiency $(\varepsilon)$, signal significance $\left(N_{s i g} / \sqrt{N_{b k g}}\right.$ with arbitrary normalization of the signal), and expected upper limit (UL) at $90 \%$ confidence level at $1 \mathrm{ab}^{-1}$ for "BGx0" and "BGx1" configurations. The errors reported and the ones used in the upper limit estimation are statistical only.

theoretically clean due to the absence of long-distant hadronic effects. Experimental searches have been performed with the BaBar and Belle data with both hadronic and semileptonic tag [5] and no evidence for signal has been found. In the left plot of Fig. 2 the experimental upper limits and the SM expectations [6] are shown. The upper limits are less than 1 order of magnitude away from SM predictions. An analysis on Belle II full simulation using hadronic tag B reconstruction and selecting $K^{*+} \rightarrow K^{+} \pi^{0}$ to establish the machine background impact has been performed. After applying selection criteria on kinematics and event-shape variables, signal efficiency and background yield have been estimated in a signal region of the extra neutral energy recorded in the electromagnetic calorimeter, associated neither to the tag side nor to the signal side. Analysis results on samples of simulated physics events superimposed on nominal machine background ("BGx1" configuration) and on machine background-free samples ("BGx0" configuration) have been compared. Negligible impact of machine background both in terms of variable distribution and signal significance has been observed, as shown in the table reported in Fig 2 and in distributions in Fig 3. Extrapolation on full Belle II statistics of the Belle hadronic and semileptonic tag analyses, assuming two times better tag $B$ reconstruction efficiency, shows that the $5 \sigma$ observation will be feasible with about $18 \mathrm{ab}^{-1}$. The predicted precision, incorporating both statistical and systematic effects, with the full statistics is of the order of $10 \%$ for all charged and neutral $K^{(*)} v \bar{v}$ channels. The fraction of longitudinally polarized $K^{*}$, helpful in distinguishing among different NP scenarios, may also be measured with full statistics at the $20 \%$-level precision.

\section{Conclusions}

As discussed in this contribution, Belle II will be a unique environment to study $B$ decays with missing energy in the final state, sensitive to indirect NP effects. Belle II will have a factor of 40 in luminosity (and much higher machine background) with respect to the first generation B-factories, BaBar and Belle. Full simulation studies, with the current knowledge of the machine background rates, have proven the detector performance and the reconstruction algorithms to be robust against 

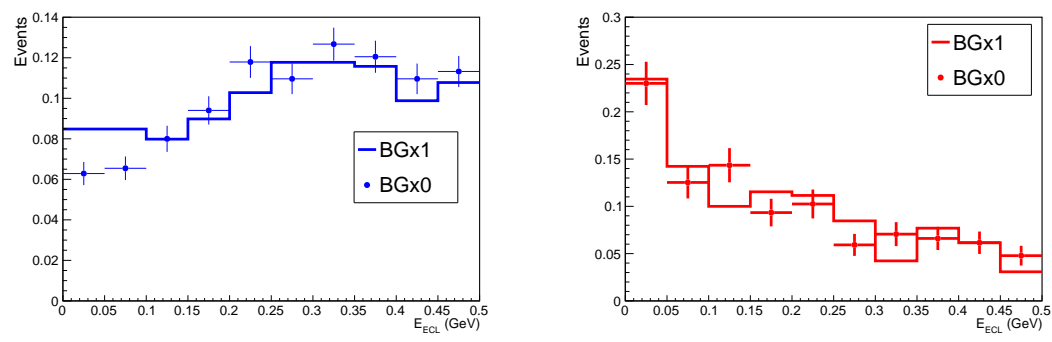

Figure 3: Extra neutral energy distribution for $B^{+} B^{-}$generic decay sample (left) and signal MC sample (right) for simulated events not superimposed to machine background (crosses) and simulated events superimposed to the nominal machine background (full histogram).

machine background. Improvements on the analysis strategy and larger data samples make Belle II sensitive to a first observation of $B \rightarrow K^{(*)} v \bar{v}$ assuming it occurs at, or higher than the rate predicted in the SM. Belle II is also uniquely positioned to further investigate deviations from or consistency with the SM predictions in the case of the $B \rightarrow \tau \nu$ and $B \rightarrow D^{(*)} \tau \nu$ channels. A detailed discussion of the Belle II detector and machine background measurements, software tools, and physics program will be published in 2017 in the "Belle II physics book".

\section{References}

[1] H. Na et al. [HPQCD Coll.], $B \rightarrow D l v$ form factors at nonzero recoil and extraction of $\left|V_{c b}\right|$, Phys. Rev. D 92 (2015) 054510 Erratum: [Phys. Rev. D 93 (2016) 119906] [hep-lat/ 150503925 ]; E. T. Neil et al. [Fermilab Lattice and MILC Coll.], Leptonic B- and D-Meson Decay Constants with 2+1 Flavors of Asqtad Fermions, PoS LATTICE 2014 (2015) 383 [hep-lat/1501.01991]; S. Fajfer et al., Search for new physics in $B \rightarrow D^{*} \tau \bar{v}_{\tau}$ decay, AIP Conf. Proc. 1492 (2012) 82.

[2] S. Hirose et al. [Belle Coll.], Measurement of the $\tau$ lepton polarization and $R\left(D^{*}\right)$ in the decay $\bar{B} \rightarrow D^{*} \tau^{-} \bar{v}_{\tau}$, Phys. Rev. Lett. 118 (2017) 211801 [hep-ex/1612.00529].

[3] C. Patrignani et al. [Particle Data Group], Review of Particle Physics, Chin. Phys. C 40 (2016) 100001.

[4] B. Kronenbitter et al. [Belle Coll.], Measurement of the branching fraction of $B^{+} \rightarrow \tau^{+} v_{\tau}$ decays with the semileptonic tagging method, Phys. Rev. D 92, 051102 (2015) [hep-ex/1503.05613]; I. Adachi et al. [Belle Coll.], Evidence for $B^{-} \rightarrow \tau^{-} \bar{v}_{\tau}$ with a Hadronic Tagging Method Using the Full Data Sample of Belle, Phys. Rev. Lett. 110, 131801 (2013) [hep-ex/1208 . 4678; J. P. Lees et al. [BaBar Coll.], Evidence of $B^{+} \rightarrow \tau^{+} v$ decays with hadronic B tags, emphPhys. Rev. D 88 (2013) 031102 [hep-ex/1207.0698]; B. Aubert et al. [BaBar Coll.], A Search for $B^{+} \rightarrow \tau^{+} v$ with Hadronic B tags, Phys. Rev. D 77 (2008) 011107 [hep-ex/ 0708 . 2260].

[5] J. Grygier et al. [Belle Coll.], Search for $B \rightarrow h v \bar{v}$ decays with semileptonic tagging at Belle, [hep-ex/1702.03224]; O. Lutz et al. [Belle Coll.], Search for $B \rightarrow h^{(*)} v \bar{v}$ with the full Belle $\Upsilon(4 S)$ data sample, Phys. Rev. D 87 (2013) 111103 [hep.ex/1303.3719]; J. P. Lees et al. [BaBar Coll.], Search for $B \rightarrow K^{(*)} v \bar{v}$ and invisible quarkonium decays, Phys. Rev. D 87 (2013) 112005 [hep-ex/1303.7465].

[6] D. M. Straub, BELLE2-MEMO-2016-007 (2016); A. J. Buras et al., $B \rightarrow K^{(*)} v \bar{v}$ decays in the Standard Model and beyond, JHEP 1502 (2015) 184 [hep-ph/1409.4557]. 